Conference Article

\title{
Synchronization of Chaotic Nonlinear Circuits via a Memristor
}

\author{
Ch. K. Volos ${ }^{*}{ }^{* 1}$, I. M. Kyprianidis ${ }^{1}$, I. N. Stouboulos ${ }^{1}$, J. M. Muñoz-Pacheco ${ }^{2}$ and V.-T. Pham ${ }^{3}$ \\ ${ }^{1}$ Department of Physics, Aristotle University of Thessaloniki, Thessaloniki, GR-54124, Greece. \\ ${ }^{2}$ Faculty of Electronics Science, Meritorious Autonomous University of Puebla, C.P. 72000, Mexico. \\ ${ }^{3}$ School of Electronics and Telecommunications, Hanoi University of Science and Technology, Vietnam.
}

Received 29 September 2014; Revised 14 October 2014; Accepted 25 October 2014

\begin{abstract}
In this work, the case of coupling between chaotic nonlinear circuits via a memristor, is studied. As a circuit, the most well-known nonlinear circuit, the circuit of Chua oscillator, is chosen. The two identical circuits are coupled unidirectionally via the HP's memristor, by using a recently new proposed window function. The simulation results show a variety of dynamical phenomena, such as chaotic synchronization and on-off intermittency, depending on memristor's initial state and the parameters of the chosen window function.
\end{abstract}

Keywords: Chaos, Chua's oscillator circuit, memristor, unidirectional coupling, complete synchronization.

\section{Introduction}

The last decades, chaotic systems have received much attention due to their potential applications in many scientific fields [1]. These systems revealed that despite of the knowledge of their evolution rules and initial conditions, their future seemed to be arbitrary and unpredictable. That opened quite a revolution in modern physics, terminating with Laplace's ideas of casual determinism [2].

Until now, chaos has been observed in weather and climate [3], population growth in ecology [4], economy [5], to mention only a few examples. It also has been observed in the laboratory in a number of systems such as electrical circuits [6], lasers [7], chemical reactions [8], fluid dynamics [9], robotics [10,11], mechanical systems, and magneto-mechanical devices [12]. So, chaos theory provides the means to explain various phenomena in nature and makes use of chaotic dynamical systems in many different scientific fields. In this direction, nonlinear electronic circuits have been used extensively for modelling and simulating the dynamic behavior of interdisciplinary nonlinear systems.

Recently, Hewlett-Packard researchers have realized a solid-state thin film two-terminal memristor [13]. After this realization, a considerable number of publications has presented noticeable results in models of memristor, fabricated materials and techniques or important applications of memristor such as high-speed low-power processors [14], adaptive filter [15], associative memory [16-18], neural networks [19] and programmable analog integrated circuits [20].

Professor L. O. Chua proposed firstly the definition of memristor in 1971 [21]. Memristor was introduced as the fourth basic circuit element besides the three classical elements, the resistor $(R)$, the inductor $(L)$ and the capacitor (C). This fourth circuit element is defined in terms of a relationship between two fundamental circuit variables, the charge $(q)$ and the flux $(\varphi)$. A charge-controlled memristor is described by

$v_{M}=M(q) i_{M}$

where $\left(v_{M}\right)$ is the voltage across the memristor and $\left(i_{M}\right)$ is the current through the memristor (Fig.1(a)). Here the memristance $(M)$ is defined by

$M(q)=\frac{d \varphi(q)}{d q}$

The flux-controlled memristor is given by

$i_{M}=W(\varphi) v_{M}$

where $W(\varphi)$ is the memductance, which is defined by

* E-mail address: volos@physics.auth.gr ISSN: 1791-2377 @ 2015 Kavala Institute of Technology. All rights reserved.

$W(\varphi)=\frac{d q(\varphi)}{d \varphi}$ 
Moreover, the nonlinear dynamical systems, called memristive systems, were introduced by generalizing the original definition of a memristor [22]. A memristive system is given as:

$$
\left\{\begin{array}{l}
\dot{x}=f(x, u, t) \\
y=g(x, u, t) u
\end{array}\right.
$$

where $(u),(y)$, and $(x)$ denote the input, output and state of the memristive system, respectively. The function $f$ is a continuous n-dimensional vector function and $g$ is a continuous scalar function.

The fingerprint of a memristor is the pinched hystereris loop as shown in Fig.1(b), which demonstrates the relationship between the voltage across the memristor $\left(v_{M}\right)$ and the current $\left(i_{M}\right)$ flowing into it by applying an external bipolar period signal, i.e, a sinusoidal signal [23]. The pinched hystereris loop shrinks when the frequency of the applied signal is adequately large.

So, in this paper the study of the effect of using an HP memristor in the coupling of two nonlinear circuits is presented. As a nonlinear circuit the most well-known circuit, the circuit of Chua oscillator, is selected. The two identical circuits are coupled unidirectionally via the proposed memristor, in which a recently new proposed window function has been used. The simulation results show a variety of dynamical phenomena, such as chaotic synchronization and on-off intermittency, depending on memristor's initial state, the chosen window function and the coupling mode. The phenomenon of full chaotic synchronization, in which the interaction between two identical coupled chaotic systems leads to a perfect coincidence of their chaotic trajectories $\left(x_{1}(t)=x_{2}(t)\right.$, as $t \rightarrow \infty)$, plays an important role, especially in the case of coupling via a memristor [24-26].

The rest of the paper is organized as follows. The next Section provides a brief description of the memristor proposed by HP scientists, which consists the base of this work. Section 3 presents the unidirectional coupling schemes, via the proposed memristor, between two identical coupled circuits of Chua oscillators. The simulation results of system's dynamic behavior are studied in details in Section 4. Finally, Section 5 outlines the conclusions that have been reached with this research study.

\section{Properties and Formulas of HP's Memristor}

After 36 years of Chua's hypothesis about memristor, this element came into life when researchers of HP lab reported the first ever solid state version of memristor in their famous article [13]. This memristor, is made of a titanium dioxide layer which is located between two platinum electrodes (Fig.2(a)). This layer is of the dimension of several nanometers and if an oxygen dis-bonding occurs, its conductance will rise instantaneously. However, without doping, the layer behaves as an isolator. The area of oxygen dis-bonding is referred to as space-charge region and changes its dimension if an electrical field is applied. This is done by a drift of the charge carriers. The smaller the insulating layer, the higher the conductance of the memristor. Also, the tunnel effect plays a crucial role.
Without an external influence the extension of the spacecharge region does not change.

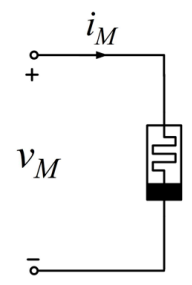

(a)

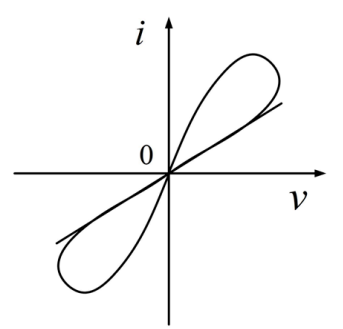

(b)

Fig. 1. Memristor and its typical "pinched hysteresis loop" when driven by a bipolar period signal input.

The internal state $(x)$ is the extent of the space-charge region, which is restricted in the interval $[0,1]$ and can be described by the equation:

$x=\frac{w}{D}, \quad 0 \leq x \leq 1, \quad x \in \mathrm{R}$

where $(w)$ is the absolute extent of the space-charge region and $(D)$ is the absolute extent of the titanium dioxide layer. The memristance can be described by the following equation:

$$
M(x)=R_{\mathrm{ON}} x+R_{\mathrm{OFF}}(1-x)
$$

where $\left(R_{\mathrm{ON}}\right)$ is the resistance of the maximum conducting state and $\left(R_{\mathrm{OFF}}\right)$ represents the opposite case (Fig.2(b)). So, when $x=0, R=R_{\mathrm{OFF}}$, and when $x=1, R=R_{\mathrm{ON}}$. The vector containing the internal states of the memristor is one dimensional. For this reason scalar notation is used. The state equation is:

$$
\frac{\mathrm{d} x}{\mathrm{~d} t}=\frac{\mu_{v} R_{\mathrm{ON}}}{D^{2}} i(t)
$$

where $\left(\mu_{v}\right)$ is the oxygen vacancy mobility and $i(t)$ is the current through the device. By using the Eq.(6) the previous equation can be rewritten as:

$$
\frac{\mathrm{d} w}{\mathrm{~d} t}=\frac{\mu_{v} R_{\mathrm{ON}}}{D} i(t)
$$

So, the dynamics of the memristor can therefore be modeled through the time dependence of the width $(w)$ of the doped region. Integrating Eq.(9) with respect to time, we have

$$
w=w_{0}+\frac{\mu_{v} R_{\mathrm{ON}}}{D} q(t)
$$


where $\left(w_{0}\right)$ is the initial width of the doped region at $t=0$ and $(q)$ is the amount of charges that have passed through the device. Substituting (6), (10) into equation (7) gives:

$$
M(q)=R_{0}-\frac{\mu_{v} R_{\mathrm{ON}} \Delta R}{D^{2}} q(t)
$$

where

$$
R_{0}=R_{\mathrm{ON}} \frac{w_{0}}{D}+R_{\mathrm{OFF}}\left(1-\frac{w_{0}}{D}\right)
$$

and $\Delta R=R_{\mathrm{OFF}}-R_{\mathrm{ON}}$. The term $\left(R_{0}\right)$ refers to the net resistance at $t=0$ that serves as the device's memory. This term is associated with the memristive state, which is essentially established through a collective contribution, i.e. it depends directly on the amount of all charges that have flown through the device. That is why, we can say that the memristor has the feature to "remember" whether it is "ON" or "OFF" after its power is turned on and off.

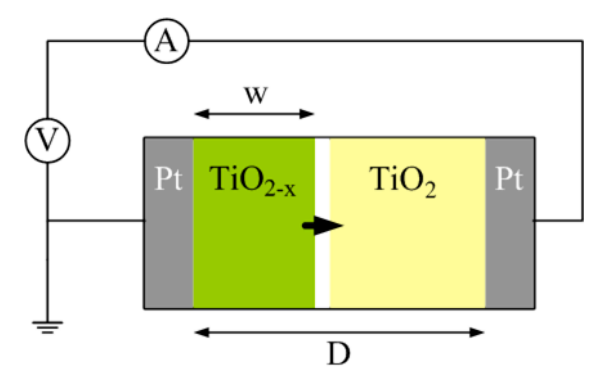

(a)

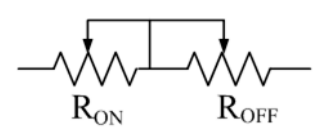

(b)

Fig. 2. (a) Structure of $\mathrm{TiO}_{2}$ memristor, in which $\mathrm{TiO}_{2-x}$ and $\mathrm{TiO}_{2}$ layers are sandwiched between two platinum electrodes, (b) equivalent circuit.

The model of memristor which has been presented by HP scientists, does not take into consideration the boundary effects as the speed of the boundary between doped and undoped regions get suppressed at either edge. For this reason, Joglekar and Wolf accounted this suppression by proposing a new window function $F(x)$ and so Eq.(9) is modified as [27]:

$$
\frac{\mathrm{d} w}{\mathrm{~d} t}=\eta \frac{\mu_{v} R_{\mathrm{ON}} i(t)}{D} F\left(\frac{w}{D}\right)
$$

where $\eta= \pm 1$ which correspond to the polarity of the memristor. The function $F(x)$ is symmetric to $x=1 / 2$ and $F(0)=F(1)=0$, for restricting ion drifting at the edge. In the interval, for $0<x<1 / 2$ the function $F(x)$ is monotonically increasing, while for $1 / 2<x<1$ is monotonically decreasing. Also, Joglekar and Wolf defined a parameter $(p)$ to constitute a family of windows functions of the form (Fig.3):

$$
F_{p}(x)=1-(2 x-1)^{2 p}
$$

As the parameter $(p)$ increases the function $F(x)$ becomes linear, while for $p=1$ the Eq.(13) reduces to HP model. In this paper, the window function of Eq.(14) has been adopted for using in the memristor model.

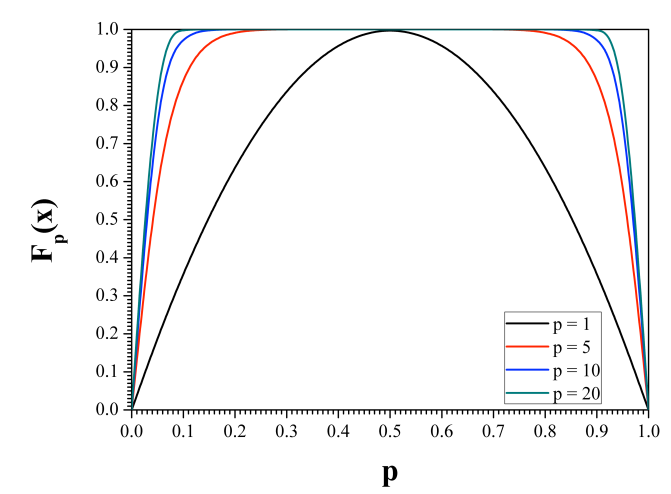

Fig. 3. The window functions of equation (14).

\section{The Coupling Scheme}

For studying the effect of the HP's memristor as a coupling element between coupled chaotic circuits the unidirectional coupling, has been used. In this coupling scheme one system drives another one and the coupling system described by the following set of differential equations [28]:

$$
\left\{\begin{array}{l}
\dot{x}_{1}=F\left(x_{1}\right) \\
\dot{x}_{2}=F\left(x_{2}\right)+C\left(x_{1}-x_{2}\right)
\end{array}\right.
$$

In this work, as a nonlinear circuit, the Chua oscillator, which is structurally the simplest and dynamically the most complex member of the Chua's circuit family, is chosen [29-31]. Until now, a great number of nonlinear phenomena concerning chaos theory have been discovered by using the specific circuit. This is the reason for choosing the Chua oscillator as the nonlinear circuit in this work.

The dynamics of the unidirectionally coupled Chua oscillators via an HP memristor (Fig.4(a)) is described by the following set of differential equations:

$$
\left\{\begin{array}{l}
\frac{\mathrm{d} x_{1}}{\mathrm{~d} \tau}=\frac{C_{0}}{C_{1}}\left[\frac{R_{\mathrm{ON}}}{R}\left(y_{1}-x_{1}\right)-f\left(x_{1}\right)\right] \\
\frac{\mathrm{d} y_{1}}{\mathrm{~d} \tau}=\frac{C_{0}}{C_{2}}\left[\frac{R_{\mathrm{ON}}}{R}\left(x_{1}-y_{1}\right)+z_{1}\right] \\
\frac{\mathrm{d} z_{1}}{\mathrm{~d} \tau}=\frac{L_{0}}{L}\left[-y_{1}-\frac{R_{0}}{R_{\mathrm{ON}}} z_{1}\right] \\
\frac{d x_{2}}{\mathrm{~d} \tau}=\frac{C_{0}}{C_{1}}\left[\frac{R_{\mathrm{ON}}}{R}\left(y_{2}-x_{2}\right)-f\left(x_{2}\right)\right] \\
\frac{\mathrm{d} y_{2}}{\mathrm{~d} \tau}=\frac{C_{0}}{C_{2}}\left[\frac{R_{\mathrm{ON}}}{R}\left(x_{2}-y_{2}\right)+z_{2}+\frac{1}{\hat{R}(\omega)}\left(y_{1}-y_{2}\right)\right] \\
\frac{\mathrm{d} z_{2}}{\mathrm{~d} \tau}=\frac{L_{0}}{L}\left[-y_{2}-\frac{R_{0}}{R_{\mathrm{ON}}} z_{2}\right] \\
\frac{\mathrm{d} \omega}{\mathrm{d} \tau}=\eta F(\omega) \frac{1}{\hat{R}(\omega)}\left(y_{1}-y_{2}\right)
\end{array}\right.
$$


where $\hat{R}(\omega)=\omega+\frac{R_{\mathrm{OFF}}}{R_{\mathrm{ON}}}(1-\omega)$ and $\eta=1$.

In system's equations, the first three equations describe the first of the two coupled Chua's oscillator, while the other three describe the second Chua's oscillator and the last one is the state equation of the proposed memristor model.

The dimensionless form of the nonlinear function $f(x)$ of the Chua's diode $N_{R}$ (Fig.4(b)) is given by the following equation:

$$
\begin{aligned}
f(x)= & m_{\mathrm{c}} x+0.5\left(m_{\mathrm{a}}-m_{\mathrm{b}}\right)(|x+1|-|x-1|)+ \\
& +0.5\left(m_{\mathrm{b}}-m_{\mathrm{c}}\right)\left(\left|x+E_{2} / E_{1}\right|-\left|x-E_{2} / E_{1}\right|\right)
\end{aligned}
$$

where, $m_{a}=R G_{a}, m_{b}=R G_{b}$ and $m_{c}=R G c$, while $G_{a}, G_{b}, G_{c}$ are the slopes of the five segments and $E_{1,2}$ are the breakpoints.

Furthermore, $x_{\mathrm{i}}, y_{\mathrm{i}}, z_{\mathrm{i}}, \omega$, and $\tau$ are the normalized parameters which are given by the following equations:

$$
x_{\mathrm{i}}=\frac{v_{\mathrm{C} 1 \mathrm{i}}}{v_{0}}, \quad y_{\mathrm{i}}=\frac{v_{C 2 \mathrm{i}}}{v_{0}}, \quad z_{\mathrm{i}}=\frac{i_{L \mathrm{i}}}{i_{0}}, \quad \omega=\frac{w}{D}, \quad \tau=\frac{t}{t_{0}}
$$

with

$v_{0}=1 \mathrm{~V}, i_{0}=\frac{v_{0}}{R_{\mathrm{ON}}}, t_{0}=\frac{D^{2}}{\mu v_{0}}, C_{0}=\frac{D^{2}}{\mu v_{0} R_{\mathrm{ON}}}, L_{0}=\frac{D^{2} R_{\mathrm{ON}}}{\mu v_{0}}$

In this work, the following values for the HP memristor have been selected: $R_{\mathrm{ON}}=100 \Omega, D=10 \mathrm{~nm}, \mu=10^{-14} \mathrm{~cm}^{2} \mathrm{~s}^{-}$ ${ }^{1} \mathrm{~V}^{-1}$. Also, the values of the coupled Chua oscillator circuits' parameters are chosen so that each coupled circuit demonstrates double-scroll chaotic attractors (Fig.4(c)), which is a signal of generating chaotic behavior [31]: $L / L_{0}=1 / 18, C_{1} / C_{0}=1 / 10, C_{2} / C_{0}=1, G=1 / R=555 \mu \mathrm{S}$, $R_{0} / R_{\mathrm{ON}}=1 / 140$. The parameter $\left(R_{\mathrm{OFF}}\right)$ plays the role of the control parameter.

\section{Simulation Results}

For studying the effect of the HP memristor as a coupling element in the unidirectional coupling system's dynamics behavior, the aforementioned window function of Eq.(14) has been used with different values of the parameter $(p)$, while the parameter $\left(R_{\mathrm{OFF}}\right)$ changes from $1 \mathrm{k} \Omega$ to $100 \mathrm{k} \Omega$. The system of differential equations (16) has been solved numerically by using the fourth order Runge-Kutta algorithm, while the initial conditions of the system are: $\left(x_{10}, y_{10}, z_{10}, x_{20}, y_{20}, z_{20}, \omega_{0}\right)=(0.8,-0.2,0.4,-0.5,0.1,0.2,0.5)$.

As it is mentioned, the function of Eq.(14) is used in the proposed memristor model. Also, the chosen value of the parameter $(p)$ is equal to one $(p=1)$, while the resistance $R_{\mathrm{OFF}}=1 \mathrm{k} \Omega$ (Fig.5). As it can been shown in Fig.5(a) the state variable $(\omega)$ becomes equal to one for $t=3.305 \mathrm{~s}$. Also, each one of the coupled circuits begins again from a chaotic double-scroll behavior, but the first circuit remains always in this mode, because it is not influenced by the other circuit, whose behavior is suppressed into single-scroll attractors around the two different equilibrium points. Finally, after a transition period, in which the system is in a chaotic desynchronization mode (Fig.5(e)), the system results in a chaotic synchronization mode (see Fig.5(b) \& (f), for $t>6.25 \mathrm{~s}$ ), in which each one of the two coupled circuits returning again in a chaotic double-scroll state (see Figs.5(c) $\&$ (d) for $t>6.25 \mathrm{~s}$ ).

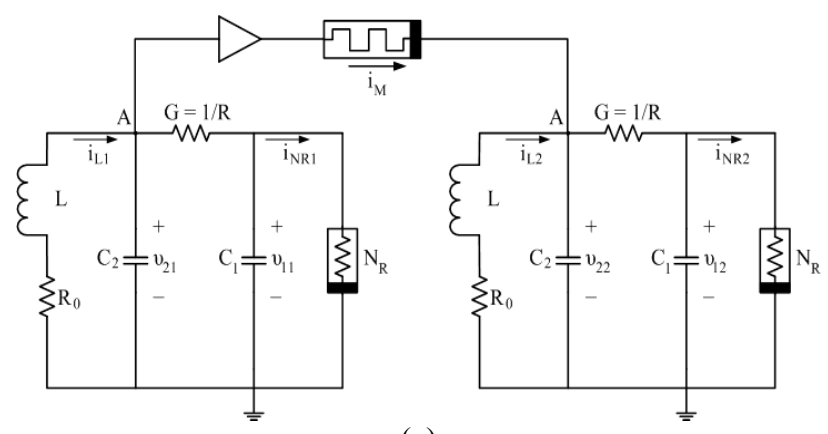

(a)

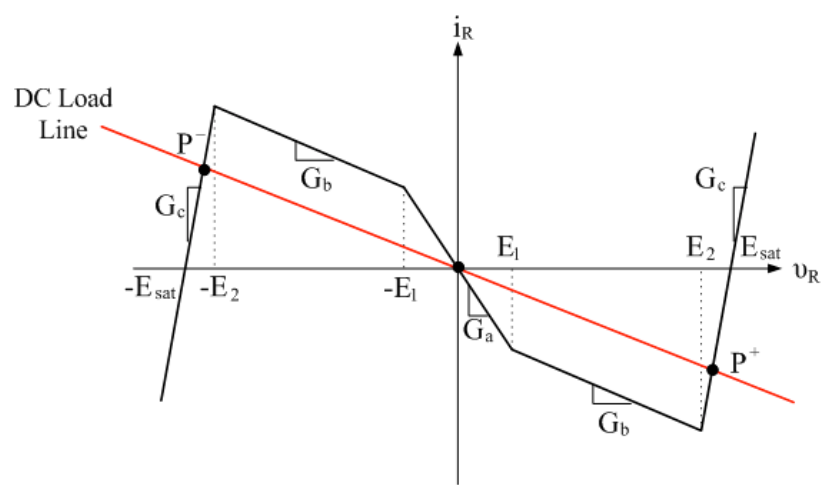

(b)

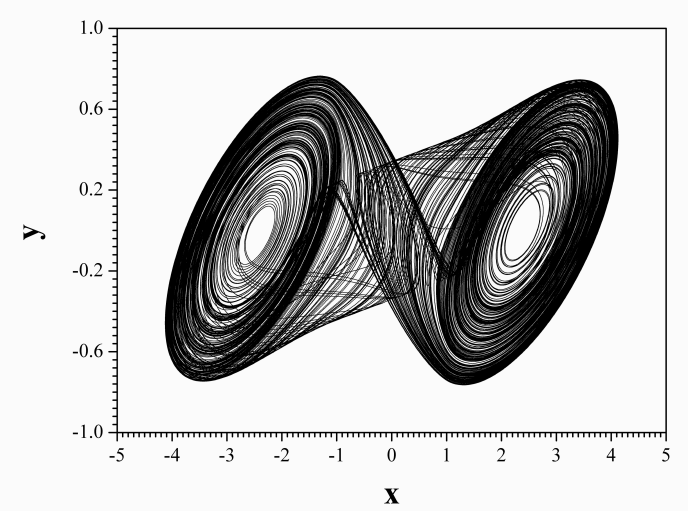

(c)

Fig. 4. (a)The schematic of unidirectionally coupled Chua oscillators, via an HP memristor, (b) the five-segment $v-i$ characteristic of the nonlinear element $N_{R}$ and (c) the projection on the $x-y$ plane of the double-scroll chaotic attractor of the Chua oscillator.

For $R_{\mathrm{OFF}}=10 \mathrm{k} \Omega$ and $p=1$, the system presents the same behavior, but the time in which the state variable $(\omega)$ becomes equal to one has been increased significantly, as it can be shown in Fig.6(a). However, after a long period of time, in which the memristor remains in this mode, a sudden removal to a chaotic oscillation mode occurs. As a result, the coupled system presents the well-known phenomenon of intermittency (Fig.6(b)). That is, in this case the system underwent a transition from desynchronization (Fig.6(c)) to incomplete synchronization, ending to full synchronization (Fig.6(d)). The dynamics associated to this transition were that of on-off intermittency [32-34].

Furthermore, for $p=1$ and greater values of $R_{\mathrm{OFF}}$ than in the previous case, the state variable $(\omega)$ of the memristor 
chaotic desynchronization mode, in which each one of the coupled circuits presents double-scroll chaotic behavior.

Finally, for a standard value of $R_{\mathrm{OFF}}(1 \mathrm{k} \Omega)$, while the parameter $(p)$ varies, an interesting alternation between two stable conditions, depending on the value of $(p)$ and the initial conditions, is observed. The boundary of the two regions and so the state variable $(\omega)$ of the memristor is moving from one state $(\omega=0)$ to the other $(\omega=1)$ and finally permanently results to one of these two limits. In more detail, for $p=\{2,8,10\}$ (Figs.7(a), (e) \& (g)) the variable $(\omega)$ tends to the lower limit $(\omega=0)$ and the system shows a permanent desynchronization state (Figs.7(b), (f), (h) \& Fig.8(b)), in which the second coupled circuit shows a double-scroll chaotic behavior (Fig.8(a)). In Fig.7(c), for $p=5$, the variable $(\omega)$ tends to the upper limit $(\omega=1)$, which means that the system shows a chaotic synchronization state interrupted by desynchronization bursts (Fig.7(d)).

So, for $p>1$ the specific window function of Eq.(14) drives the system into two different permanent states, the desynchronization or chaotic synchronization state, depending on the value of $(p)$ and the initial conditions of the system. Also, the increasing of the value of $(p)$ drives the system faster to the aforementioned state.

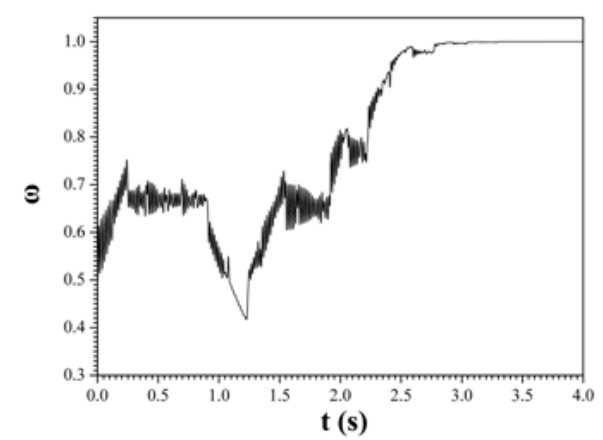

(a)

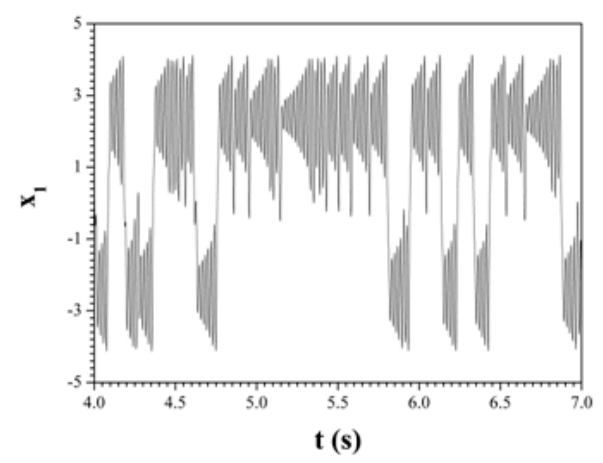

(c)

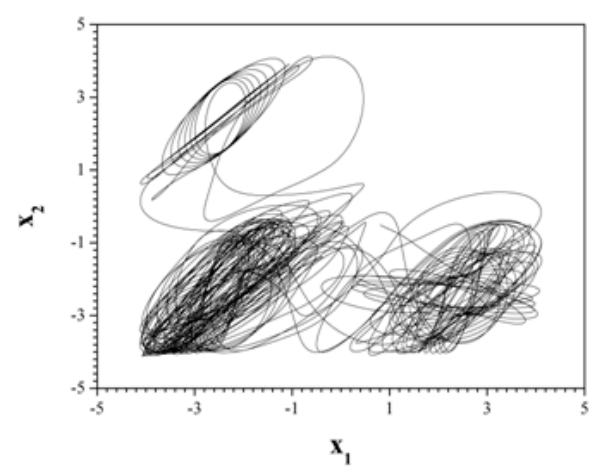

(e)

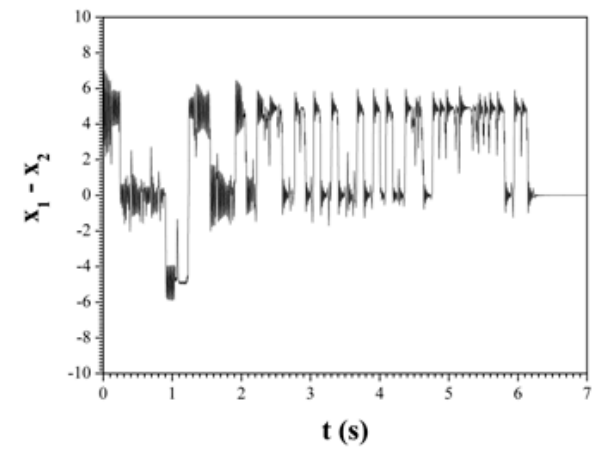

(b)

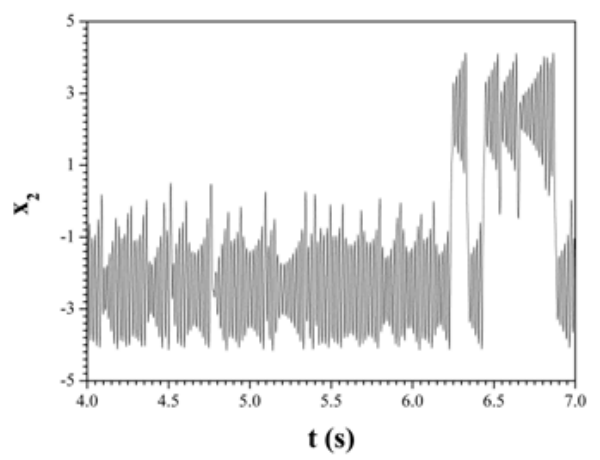

(d)

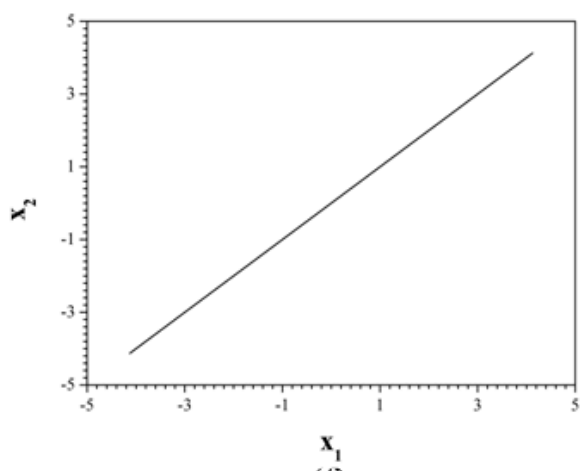

(f)

Fig. 5. Time-series of (a) the normalized variable $(\omega)$ of the width of the doped region, (b) the difference signal $\left(x_{1}-x_{2}\right)$, (c) the signal $\left(x_{1}\right)$, (d) the signal $\left(x_{2}\right)$ and phase portraits of (e) $x_{2}$ vs. $x_{1}(\mathrm{t}<2.5 \mathrm{~s})$ and (f) $x_{2}$ vs. $x_{1}(t>6.25 \mathrm{~s})$, for $R_{\mathrm{OFF}}=1 \mathrm{k} \Omega$ and $p=1$. 


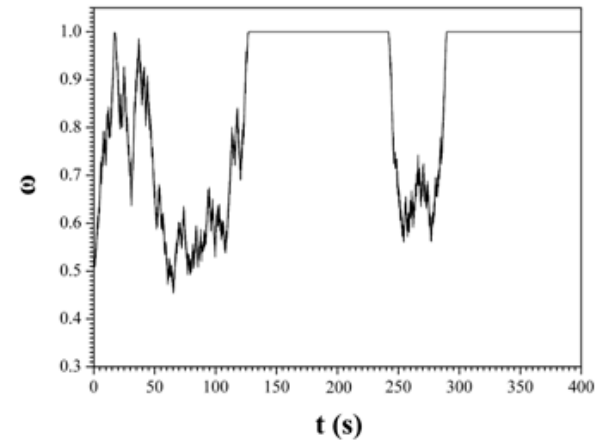

(a)

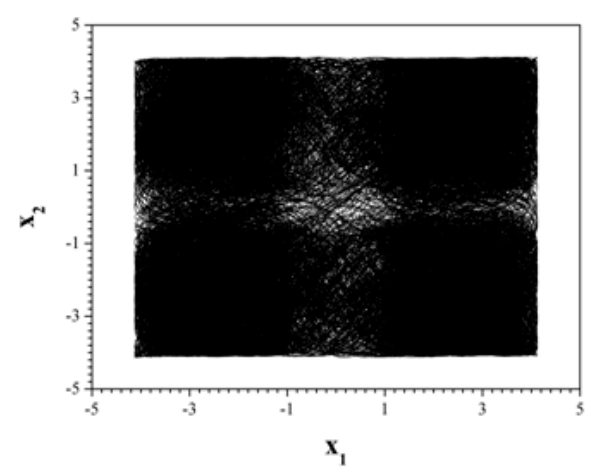

(c)

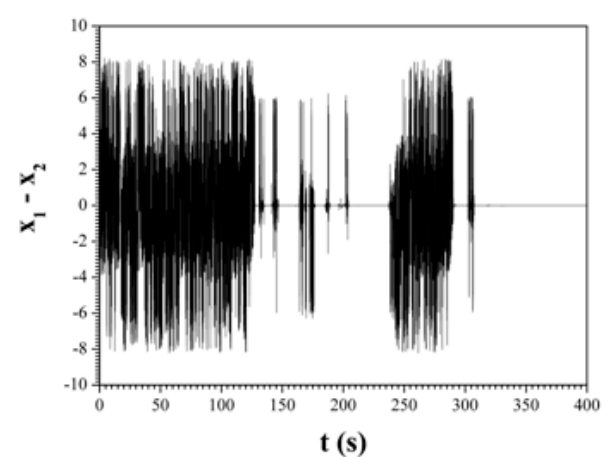

(b)

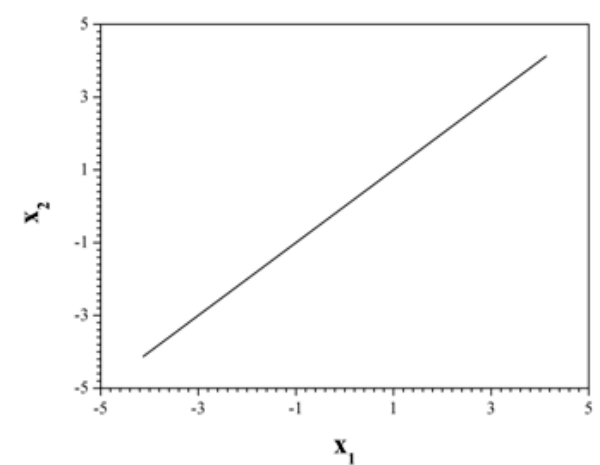

(d)

Fig. 6. Time-series of (a) the normalized variable $(\omega)$ of the width of the doped region, (b) the difference signal $x_{1}-x_{2}$ and phase portraits of (c) $x_{2}$ vs. $x_{1}(t<100 \mathrm{~s})$ and (d) $x_{2}$ vs. $x_{1}(t>350 \mathrm{~s})$, for $R_{\mathrm{OFF}}=10 \mathrm{k} \Omega$ and $p=1$.

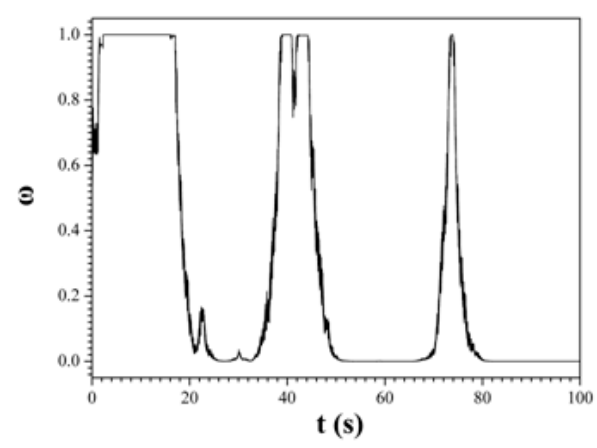

(a)

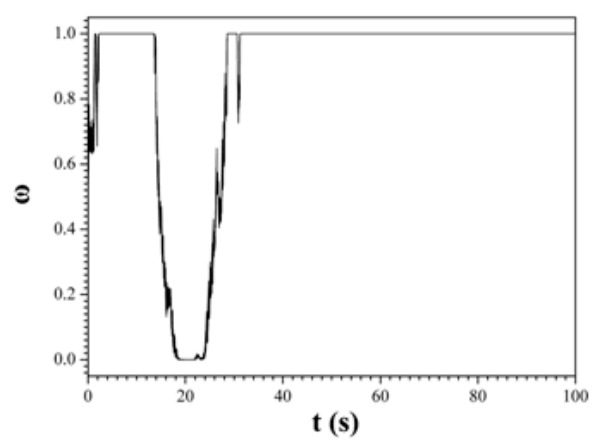

(c)

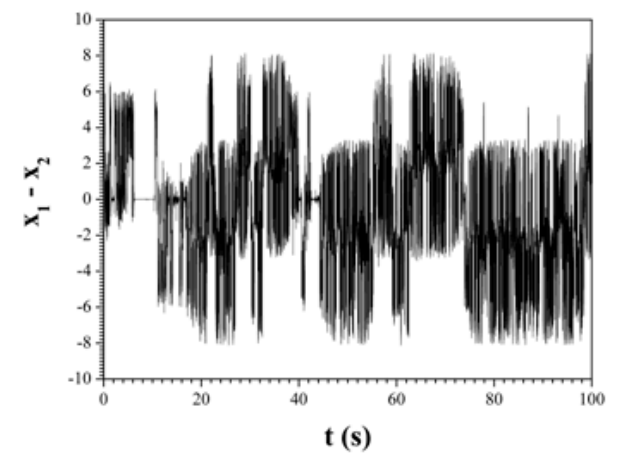

(b)

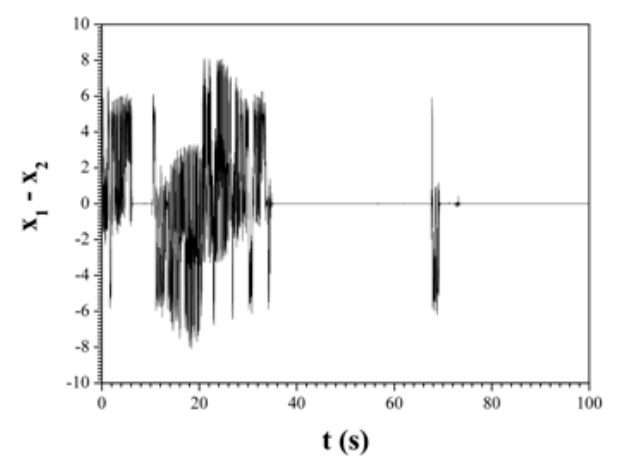

(d)

(continued) 


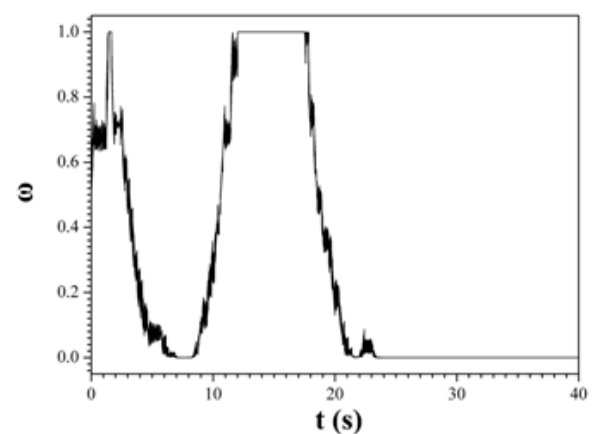

(e)

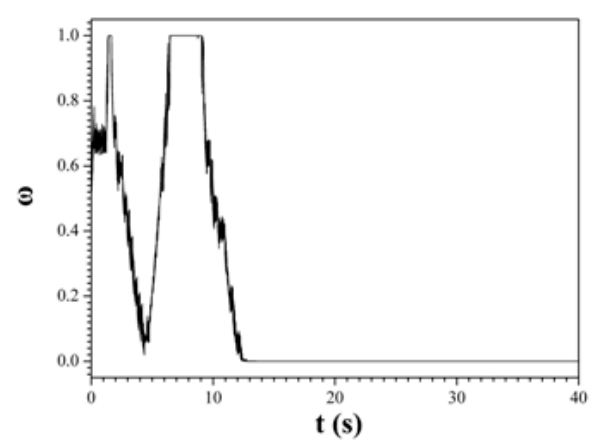

(g)

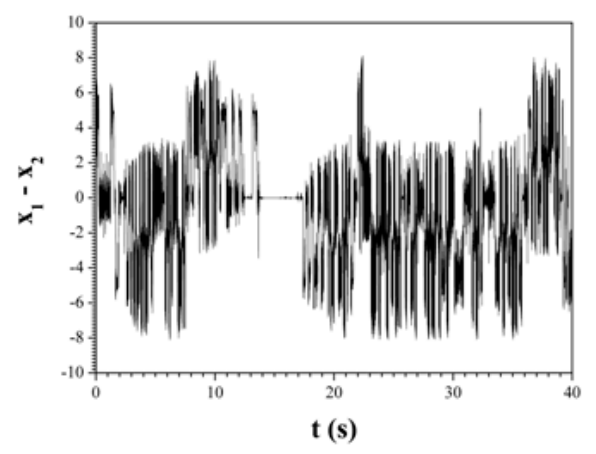

(f)

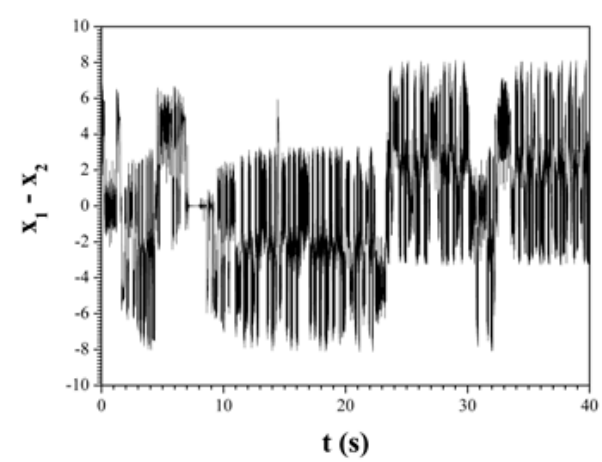

(h)

Fig. 7. Time-series of the normalized variable $(\omega)$ and the difference signal $\left(x_{1}-x_{2}\right)$, for $R_{\mathrm{OFF}}=1 \mathrm{k} \Omega$ and (a) $\&$ (b) $p=2$, (c) $\&$ (d) $p=5$, (e) \& (f) $p=8$ and (g) \& (h) $p=10$.

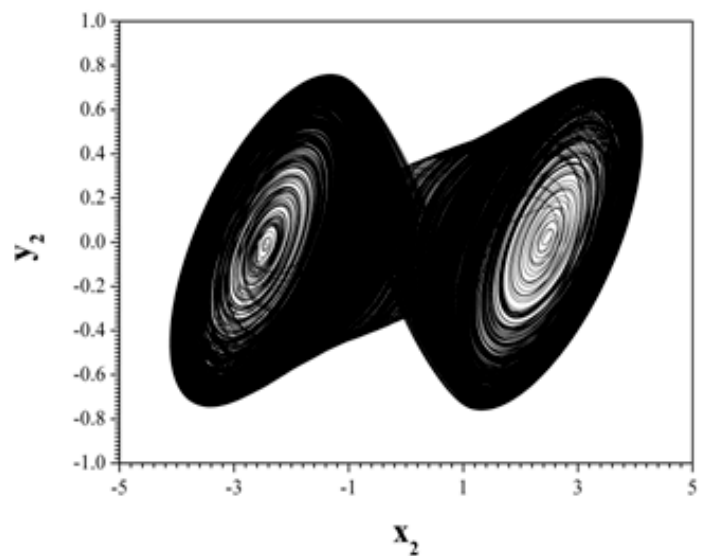

(a)

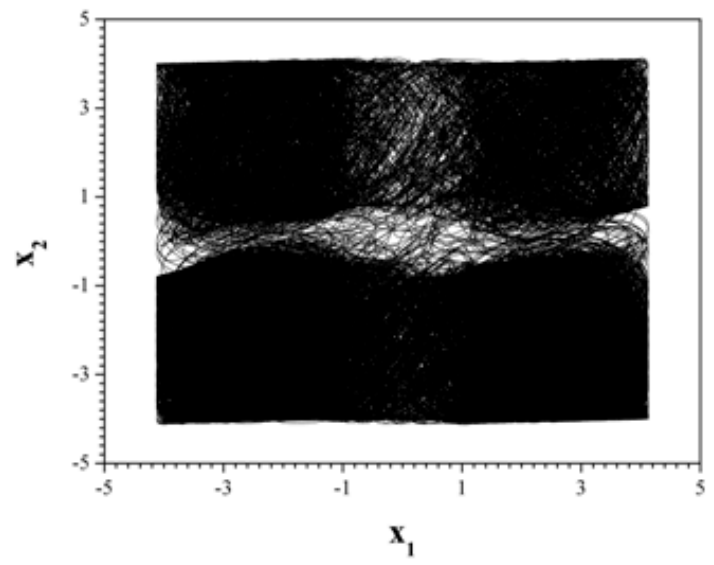

(b)

Fig. 8. Phase portraits of (a) $y_{2}$ vs. $x_{2} \&$ (b) $x_{2}$ vs. $x_{1}$, for $t>20 \mathrm{~s}$ and $p=10$.

\section{Conclusion}

In this work the case of two unidirectionally coupled identical nonlinear circuits via the proposed HP memristor was presented. As a nonlinear circuit, the most well-known, the circuit of Chua's oscillator, was chosen due to its properties. For studying the effect of the HP memristor as a coupling element in the coupling system's dynamic behavior, a recently new proposed window function has been used with different values of memristor's parameters $(p)$ and $\left(R_{\mathrm{OFF}}\right)$.

By using the window function, which was presented by Joglekar and Wolf, for taking into consideration the boundary effects, the coupling system could be in two different dynamical states, depending mainly on the values of the parameter $\left(R_{\mathrm{OFF}}\right)$. In more detail, for low values of the resistance $\left(R_{\mathrm{OFF}}\right)$ the state variable $(\omega)$ increased from its initial value and gradually reached its upper limit $(\omega \rightarrow 1)$, which means that the space-charge region $(w)$ was expanded and had the tension to cover the entire titanium dioxide layer of the memristor. As a consequence the resistance of the maximum conducting state was $R=R_{\mathrm{ON}}$ and after a long transition time the system resulted in full chaotic synchronization mode. However, before the system resulted in this state, the second of the coupled circuits had started from a chaotic double-scroll state and under the influence of 
the memristor, this behavior was suppressed into singlescroll attractor around the two circuits' equilibrium points.

For greater values of $\left(R_{\mathrm{OFF}}\right)$ the system presented the same route from chaotic desynchronization to full chaotic synchronization. The only difference was the significant greater time in which the variable $(\omega)$ became equal to one. Furthermore, after a long period of time, in which the memristor remains in this mode, a sudden removal to a chaotic oscillation mode occurred. As a result, the coupled system presented the well-known phenomenon of intermittency.

Finally, for $R_{\mathrm{OFF}}=1 \mathrm{k} \Omega$ and when the parameter $(p)$ is varied, an interesting alternation between two stable conditions, depending on the value of $(p)$ and the initial conditions, was observed. The boundary of the two regions and so the state variable $(\omega)$ of the memristor was moving from one state $(\omega=0)$ to the other $(\omega=1)$ and finally permanently resulted to one of these two limits.

\section{References}

1. S.H. Strogatz, Nonlinear dynamics and chaos: With applications to physics, biology, chemistry, and engineering, Perseus Books, Massachusetts, USA (1994).

2. P.S. Laplace, Traité du mécanique céleste. Oeuvres complètes de Laplace, Gauthier-Villars, Paris, France (1825).

3. E.N. Lorenz, Deterministic non-periodic flow, J. Atm. Sciences, vol. 20, pp. 130-141 (1963).

4. R.M. May and A.R. McLean, Theoretical ecology: Principles and applications, Blackwell, Oxford, UK (2007).

5. C. Kyrtsou and C. Vorlow, Complex dynamics in macroeconomics: A novel approach, In: New Trends in Macroeconomics; Diebolt, C. and Kyrtsou, C. ISBN-13: 978-3540-21448-9, Springer Verlag, Berlin, Germany (2005).

6. B. Van der Pol and J. Van der Mark, Frequency demultiplication, Nature, vol. 120, pp. 363-364 (1927).

7. L.W. Casperson, Gas laser instabilities and their interpretation, In Proceedings of the NATO Advanced Study Institute, Springer Verlag, Berlin, Germany (pp. 83-98) (1988).

8. R.J. Field and L. Györgyi, Chaos in chemistry and biochemistry, World Scientific Publishing, Singapore (1993).

9. G.L. Baker, Chaotic dynamics: An introduction, Cambridge University Press, Cambridge, UK (1996).

10. Ch. K. Volos, I. M. Kyprianidis, and I. N. Stouboulos, A chaotic path planning generator for autonomous mobile robots, Robotics and Autonomous Systems, vol. 60, pp. 651-656 (2012).

11. Ch. K. Volos, I. M. Kyprianidis, and I. N. Stouboulos, Motion control of robots using a chaotic truly random bits generator, Journal of Engineering Science and Technology Review, vol 5(2), pp. 6-11 (2012).

12. F.C. Moon, Chaotic vibrations: An introduction for applied scientists and engineers, Wiley, Weinheim, Germany (1987).

13. D. Strukov, G. Snider, G. Stewart, and R. Williams, The missing memristor found, Nature, vol. 453, pp. 80-83 (2008).

14. J.J. Yang, D.B. Strukov, and D.R. Stewart, Memristive devices for computing, Nature Nanotechnology, vol. 8, pp. 13-24 (2013).

15. T. Driscoll, J. Quinn, S. Klein, H. T. Kim, B. J. Kim, Y.V. Pershin, M. Di Ventra, and D.N. Basov, Memristive adaptive filters, Appl. Phys. Lett., vol. 97, pp. 093502 (2010).

16. Y.V. Pershin and M. Di Ventra, Experimental demonstration of associative memory with memristive neural networks, Neural Networks, vol. 23, p. 881 (2010).

17. L. Wang, Ch. Zhang, L. Chen, J Lai, and J. Tong, A novel memristor-based rSRAM structure for multiple-bit upsets immunity, IEICE Electronics Express, vol. 9, pp. 861-867 (2012).

18. Y. Shang, W. Fei, and H. Yu, Analysis and modeling of internal state variables for dynamic effects of nonvolatile memory devices, IEEE Trans. Circuits Sys. I: Regular Papers, vol. 59, pp. 1906-1918 (2012)

19. S.P. Adhikari, C. Yang, H. Kim, and L.O. Chua, Memristor bridge synapse-based neural network and its learning, IEEE
Transactions on Neural Networks and Learning Systems, vol. 23, pp. 1426-1435 (2012).

20. S. Shin, K. Kim, and S. M. Kang, Memristor applications for programmable analog ICs, IEEE Trans. Nanotechnology, vol. 410, pp. 266-274 (2011).

21. L.O. Chua, Memristor - the missing circuit element, IEEE Transactions on Circuit Theory, vol. 18(5), pp. 507-519 (1971).

22. L.O. Chua and S.M. Kang, Memristive devices and systems, In Proceedings of the IEEE, vol. 64, pp. 209-223 (1976).

23. S.P. Adhikari, M.P. Sad, H. Kim, and L.O. Chua, Three fingerprints of memristor, IEEE Trans. Circuits Sys. I: Regular Papers, vol. 60, pp. 3008-3021 (2013).

24. S. Banerjee, Chaos synchronization and cryptography for secure communications: Applications for encryption, IGI Global, U.S.A. (2010).

25. S. Boccaletti, J. Kurths, G. Osipov, D.L. Valladares, and C.S. Zhou, The synchronization of chaotic systems, Physics Reports, vol. 366, pp. 1-101 (2002).

26. A. Pikovsky, M. Rosenblum, and J. Kurths, Synchronization: A universal concept in nonlinear sciences, Cambridge University Press, New York, USA (2001).

27. Y.N. Joglekar and S.J. Wolf, The elusive memristor: Properties of basic electrical circuits, European Journal of Physics, vol. 30, pp. 661-675 (2009).

28. Ch. K. Volos, I. M. Kyprianidis, and I. N. Stouboulos, Experimental synchronization of two unidirectionally coupled double scroll circuits, In Proceedings of the 7 th International Conference of the Balkan Physical Union, (BPU 2009), AIP Conference Proceedings, vol. 1203, pp. 403-408, Alexandroupolis, Greece (2009).

29. L.O. Chua, C.W. Wu, A. Huang, and G.-Q. Zhong, A universal circuit for studying and generating chaos - Part I: Routes to chaos, IEEE Trans. Circuits Syst., vol. 40, pp. $732-744$ (1993).

30. L. Fortuna, M. Frasca, and M.G. Xibilia, Chua's circuit implementations: Yesterday, today and tomorrow, World Scientific, Singapore (2009).

31. C.W. Wu and L. Pivka, From Chua's circuit to Chua's oscillator: A picture book of attractors, World Scientific, Singapore (1994).

32. P.W. Hammer, N. Plat, S.M. Hammel, J. F. Heagy, and B. D. Lee, Experimental observation of on-off intermittency, Phys. Rev. Lett., vol. 73, pp. 1095-1098 (1994)

33. I.M. Kyprianidis, Ch.K. Volos, S.G. Stavrinides, I.N. Stouboulos, A. N. Anagnostopoulos, On-off intermittent synchronization between two bidirectional coupled double scroll circuits, Commun. Nonlinear Sc. Numer. Simulat., vol. 15, pp. 2192-2200 (2010).

34. N. Plat, E.A. Spiegel, and C. Tresser, On-off intermittency: a mechanism for bursting, Phys. Rev. Lett., vol. 70, pp. 279-282 (1993). 